Table 1. Baseline demographic and clinical characteristics, cardiovascular risk factors and treatments received.

\begin{tabular}{|c|c|c|c|c|c|c|c|}
\hline Variables & $\begin{array}{l}\text { All patients } \\
n=200 \\
\text { median (IQR) or } n(\%)\end{array}$ & $\begin{array}{l}\text { CVD -ve, } \\
\mathrm{n}=170 \\
\text { median (IQR) } \\
\text { or } \mathrm{n}(\%)\end{array}$ & $\begin{array}{l}\text { CVD +ve, } \\
\mathrm{n}=30 \\
\text { median (IQR) } \\
\text { or } \mathrm{n}(\%)\end{array}$ & $p$-value & $\begin{array}{l}\text { NSAID -ve, } \mathrm{n}=61 \\
\text { median (IQR) } \\
\text { or } \mathrm{n}(\%)\end{array}$ & $\begin{array}{l}\text { NSAID +ve, } n=139 \\
\text { median (IQR) or } n(\%)\end{array}$ & $p$-value \\
\hline Male, n (\%) & $119(59.5 \%)$ & $100(58.6 \%)$ & $19(63.3 \%)$ & 0.228 & $39(63.9 \%)$ & $80(57.6 \%)$ & 0.397 \\
\hline Age, years & $47.5(40.0-56.0)$ & $46.5(37.7-54.0)$ & $57.0(45.3-65.8)$ & $<0.001^{\star}$ & $49.0(44.0-56.5)$ & $46.0(38.0-54.8)$ & 0.176 \\
\hline Disease duration, years & $4.3(1.8-7.9)$ & $4.1(1.7-7.0)$ & $6.0(2.1-8.6)$ & 0.066 & $4.6(1.5-8.7)$ & $4.3(1.9-7.3)$ & 0.393 \\
\hline Diabetes, $\mathrm{n}(\%)$ & $45(22.5 \%)$ & $30(17.6 \%)$ & $15(50.0 \%)$ & $<0.001^{*}$ & $10(16.4 \%)$ & $35(25.2 \%)$ & 0.171 \\
\hline Hypertension, n (\%) & 68 (34.0\%) & $46(27.1 \%)$ & 22 (73.3\%) & $<0.001^{*}$ & 21 (34.4\%) & 47 (33.9\%) & 0.933 \\
\hline CRP, mg/L & $4.9(1.7-12.6)$ & $4.2(1.5-12.0)$ & $11.3(2.4-19.6)$ & $0.035^{\star}$ & $5.5(1.7-15.1)$ & $7.2(1.4-15.8)$ & 0.770 \\
\hline $\mathrm{ESR}, \mathrm{mm} / \mathrm{hr}$ & $21(10.0-38.0)$ & $20(9-35)$ & $31(14-60)$ & $0.038^{*}$ & $21(7-33)$ & $21(11-43)$ & 0.291 \\
\hline Systolic blood pressure, $\mathrm{mmHg}$ & $125(115-140)$ & $124(115-137)$ & $144(129-160)$ & $<0.001^{*}$ & $123(118-137)$ & $125(115-141)$ & 0.889 \\
\hline Diastolic blood pressure, $\mathrm{mmHg}$ & $78(70-85)$ & $78(70-84)$ & $82(72-90)$ & 0.199 & $78(72-86)$ & $78(70-85)$ & 0.697 \\
\hline FRS & $8.4(4.0-17.0)$ & $7.5(3.3-14.0)$ & $19.6(13.4-43.0)$ & $<0.001^{*}$ & $7.9(3.5-15.5)$ & $8.7(4.2-17.1)$ & 0.885 \\
\hline Lipid-lowering drugs, $n$ (\%) & $30(15.0 \%)$ & $25(14.7 \%)$ & $5(16.7 \%)$ & 0.782 & $9(14.8 \%)$ & $21(15.1 \%)$ & 0.949 \\
\hline MTX, n (\%) & 99 (49.5\%) & 81 (47.6\%) & $18(60.0 \%)$ & 0.212 & $25(41.0 \%)$ & $74(53.2 \%)$ & 0.111 \\
\hline bDMARDs, n (\%) & $17(8.5 \%)$ & $13(7.6 \%)$ & $4(13.3 \%)$ & 0.303 & $6(9.8 \%)$ & $11(7.9 \%)$ & 0.654 \\
\hline NSAIDs, n (\%) & $139(69.2 \%)$ & $119(70.0 \%)$ & $20(66.7 \%)$ & 0.715 & N/A & N/A & N/A \\
\hline Steroid, n (\%) & $2(1.0 \%)$ & $2(1.2 \%)$ & $0(0 \%)$ & 1.000 & $1(1.6 \%)$ & $1(0.7 \%)$ & 0.518 \\
\hline
\end{tabular}

*statistically significant at $p \leq 0.05 C V D+v e$, patients who developed cardiovascular events during subsequent follow-up;CVD-ve, patients who did not developed cardiovascular events during subsequent follow-up;NSAIDs, nonsteroidal anti-inflammatory drugs; CRP, C-reactive protein; ESR, erythrocyte sedimentation rate; FRS, Framingham Risk Score; MTX, methotrexate; bDMARDs, biological disease-modifying antirheumatic drugs.

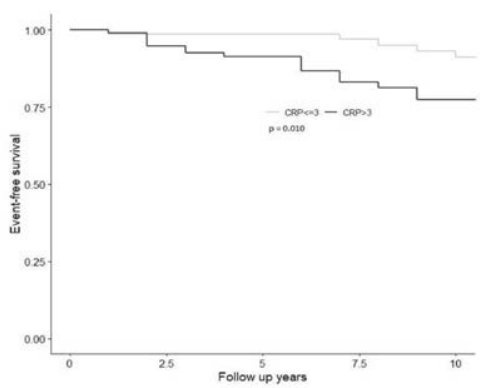

Figure IA

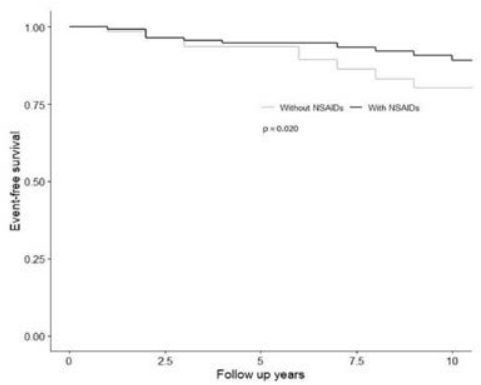

Figure 1B

Figure 1. Kaplan-Meier curves showing the cardiovascular event-free survival between patients with A) CRP 3 mg L and CRP 3 mg L; B) treated with NSAIDs and without NSAIDs use during their follow-up intervals.

Conclusion: Increased inflammatory burden as reflected by elevated CRP level was associated with increased risk of CV events, while the risk was significantly reduced with NSAIDs use in PsA patients.

Disclosure of Interests: None declared.

DOI: 10.1136/annrheumdis-2021-eular.3118

\section{POS1080 \\ HYPERLIPIDEMIA IN PSORIATIC DISEASE: HIGHER PREVALENCE IN PSORIATIC ARTHRITIS AND INVERSE ASSOCIATION WITH SYSTEMIC THERAPY}

R. Queiró Silva ${ }^{1}$, E. Pardo Campo ${ }^{1}$, S. Fernández ${ }^{1}$, L. Arboleya Rodríguez ${ }^{1}$, I. Zapico ${ }^{1}$, F. Jirout ${ }^{1} .{ }^{1}$ Hospital Universitario Central de Asturias, Rheumatology, OVIEDO, Spain

Background: Although the evidence that testifies to a higher prevalence of cardiometabolic risk factors in psoriatic disease is robust, there are not too many studies that have analyzed which disease traits are associated with these risk factors both in psoriasis and psoriatic arthritis ${ }^{1}$.

Objectives: We aimed to analyze the frequency and disease-associated features of hyperlipidemia in psoriatic disease.

Methods: Cross-sectional observational study that included 290 patients with psoriatic arthritis (mean age $54 \pm 12$ years, $54.8 \%$ male, psoriasis average duration $21 \pm 10$ years) and 310 with psoriasis alone (mean age $53 \pm 11.5$ years, $52.9 \%$ male, psoriasis average duration $22 \pm 11$ years). We first analyzed the frequency of this comorbidity and then the factors associated with it using conditional logistic regression. The significant factors in this first model were introduced in a multivariate model using a backward stepwise approach.

Results: Main disease features are depicted in Table 1. A total of 124 patients had hyperlipidemia (20.7\%). Among arthritis patients, 82/290 (28.3\%) had hyperlipidemia, compared with $42 / 310$ (13.5\%) of those with psoriasis alone (OR 2.5 , $95 \% \mathrm{Cl}: 1.7-3.3, p<0.001)$. Hyperlipidemia was independently associated with age [OR $1.07,95 \% \mathrm{Cl}: 1.04-1.11, \mathrm{p}<0.001]$ and systemic therapy [OR $0.4,95 \% \mathrm{Cl}$ $0.17-0.89, p=0.026]$.

Conclusion: Hyperlipidemia is common in psoriatic disease, but much more among arthritis patients. This comorbid factor seems to go in parallel with increasing age. There is an inverse association between systemic treatment and hyperlipidemia that should be endorsed with well-designed longitudinal studies. REFERENCES:

[1] Puig L. Cardiometabolic comorbidities in psoriasis and psoriatic arthritis. Int J Mol Sci 2018; 19: 58

Table 1. Disease characteristics of both subpopulations

\begin{tabular}{|c|c|c|c|}
\hline Variable & $\begin{array}{l}\text { PsA } \\
n=290\end{array}$ & $\begin{array}{l}\text { Psoriasis } \\
n=310\end{array}$ & $\mathrm{p}$-values \\
\hline Age $(y r \pm S D)$ & $54 \pm 12$ & $53 \pm 11.5$ & NS \\
\hline Age at psoriasis onset $(\mathrm{yr} \pm \mathrm{SD})$ & $32 \pm 16$ & $31 \pm 14.2$ & NS \\
\hline Age at arthritis onset $(\mathrm{yr} \pm \mathrm{SD})$ & $46 \pm 14$ & & NS \\
\hline Duration of psoriasis $(\mathrm{yr} \pm \mathrm{SD}$ ) & $21 \pm 10$ & $22 \pm 11$ & NS \\
\hline Duration of arthritis $(\mathrm{yr} \pm \mathrm{SD})$ & $11 \pm 7.2$ & & NS \\
\hline Male gender $(n, \%)$ & $159(54.8)$ & $164(52.9)$ & NS \\
\hline Primary education $(\mathrm{n}, \%)$ & $145(50)$ & $148(47.7)$ & NS \\
\hline Secondary education (n, \%) & 79 (27.2) & $87(28.1)$ & NS \\
\hline University degree $(n, \%)$ & $66(22.8)$ & $235(24.2)$ & NS \\
\hline Plaque psoriasis (n, \%) & $250(86.2)$ & $272(87.7)$ & NS \\
\hline Nail disease $(n, \%)$ & $122(42.1)$ & $110(35.5)$ & NS \\
\hline Psoriasis in $\geq 3$ body areas (n, \%) & $130(45)$ & $155(50)$ & $<0.05$ \\
\hline Family history of psoriasis $(n, \%)$ & $130(45)$ & $136(44)$ & NS \\
\hline Family history of PsA $(n, \%)$ & $44(15.2)$ & $15(4.8)$ & \\
\hline PASI & $6.5 \pm 4.3$ & $6.8 \pm 3.5$ & \\
\hline Oligoarthritis (n, \%) & $122(42.1)$ & & \\
\hline Polyarthritis (n, \%) & $81(28)$ & & \\
\hline Axial disease (n, \%) & $17(5.8)$ & & \\
\hline Mixed pattern (n, \%) & $70(24.1)$ & & \\
\hline Dactylitis (n, \%) & $87(30)$ & & \\
\hline DIP joint disease (n, \%) & $72(24.8)$ & & \\
\hline Mutilating arthritis $(\mathrm{n}, \%)$ & $5(1.7)$ & & \\
\hline Erosive disease $(\mathrm{n}, \%)$ & $58(20)$ & & \\
\hline $\mathrm{HAQ}($ mean $\pm \mathrm{SD})$ & $0.74 \pm 0.32$ & & \\
\hline${ }^{*} \mathrm{BASDAl}($ mean $\pm \mathrm{SD})$ & $3.64 \pm 2.12$ & & \\
\hline Pain VAS (mean \pm SD) & $4.09 \pm 2.64$ & & \\
\hline HLA-B*27 (n, \%) & $52(17.9)$ & & \\
\hline HLA-C*06 (n, \%) & $112(38.6)$ & $124(40)$ & NS \\
\hline $\operatorname{NSAID}(\mathrm{n}, \%)$ & $72(24.8)$ & $47(15.2)$ & NS \\
\hline Glucocorticoids (n, \%) & $34(11.7)$ & $15(4.8)$ & NS \\
\hline $\operatorname{MTX}(n, \%)$ & $189(65.2)$ & $128(41.3)$ & $<0.05$ \\
\hline Biologics (n, \%) & $128(44.1)$ & $132(42.6)$ & NS \\
\hline
\end{tabular}

SD, standard deviation; PsA, psoriatic arthritis; PASI, psoriasis area and severity index; DIP, distal interphalangeal joint; $H A Q$, Health Assessment Questionnaire; BASDAI, bath ankylosing spondylitis disease activity index; VAS, visual analog scale; NSAID, non-steroidal anti-inflammatory drugs. MTX, methotrexate. ${ }^{*}$ Only in patients with axial disease

Disclosure of Interests: None declared.

DOI: 10.1136/annrheumdis-2021-eular.3170 